\title{
Mortality of Neonatal Sepsis with Abnormal Blood Glucose Level
}

\author{
Made Laksmi Dewi Adnyana*, I Gusti Ngurah Sanjaya, Made Sukmawati, Ni Putu Siadi Purniti, \\ Ida Bagus Subanada, I Wayan Gustawan
}

Department of Child Health, Faculty of Medicine, Udayana University, Denpasar, Indonesia

\section{Email address:}

madelaksmi98@gmail.com (M. L. D. Adnyana),sanjayaputra65@gmail.com (I G. N. Sanjaya), sukma_bali75@yahoo.co.id (M. Sukmawati), siadi@yahoo.com (N. P. S. Purniti), subanada@yahoo.com (I. B. Subanada), iwayangustawan@gmail.com (I W. Gustawan)

${ }^{*}$ Corresponding author

\section{To cite this article:}

Made Laksmi Dewi Adnyana, I Gusti Ngurah Sanjaya, Made Sukmawati, Ni Putu Siadi Purniti, Ida Bagus Subanada, I Wayan Gustawan. Mortality of Neonatal Sepsis with Abnormal Blood Glucose Level. American Journal of Pediatrics. Vol. 7, No. 1, 2021, pp. 9-13. doi: 10.11648/j.ajp.20210701.13

Received: January 26, 2021; Accepted: February 6, 2021; Published: February 10, 2021

\begin{abstract}
Sepsis is the third leading cause of death in neonates after asphyxia and low birth weight babies (LBW). Abnormal blood glucose, both hypoglycemia and hyperglycemia, were common metabolic changes in neonatal sepsis. This condition can higher the risk of morbidity and mortality in neonatal sepsis. The aim of this study is to prove the difference in mortality of neonatal sepsis with abnormal blood glucose compared to normal blood glucose. This was a cohort retrospective study with subjects were neonate with 28-42 weeks of gestational age with neonatal sepsis confirmed from clinical sign or blood culture at neonatal ward Sanglah hospital in the period January-August 2020. Subjects were classified as abnormal and normal blood glucose level. Chi-square test was used to assess the difference of mortality in blood glucose level. Multivariate analysis was performed with logistic regression. A total of 64 subjects with abnormal blood glucose and 64 subjects with normal blood glucose were included in this study. Mortality in subject with abnormal blood glucose was 50\% and $17.2 \%$ with normal blood glucose $(P<0,005)$. Multivariate analysis found abnormal blood glucose increases the risk of mortality 3,04 times compare with normal blood glucose (95\% CI 1.09 to $8,45, P<0.033)$. Mechanical ventilation and asphyxia can also increase the risk of mortality in neonatal sepsis by 12,33 times $(95 \%$ CI 3,99 to $38,07, P<0,001)$ and 8.17 times $(95 \%$ CI 2,35 to $28,37, P<0,001)$ respectively. Subanalysis found hypoglycemia increases the risk of mortality 3.75 times compare to normoglycemia $(95 \% \mathrm{CI}$ 2.06 to $6.82, P<0.005)$, and hyperglycemia increases the risk of mortality 2.12 times compare to normoglycemia (95\% CI 1.04 to 4.26, $P=0.035)$. Conclusion there are significant difference of mortality in neonatal sepsis with abnormal blood glucose which is higher than normal blood glucose level. This research also found mechanical ventilation and asphyxia can effect the mortality in neonatal sepsis.
\end{abstract}

Keywords: Neonate, Abnormal Blood Glucose Level, Neonatal Sepsis

\section{Introduction}

Abnormal sugar level was common metabolic changes in neonatal sepsis and indirectly increase the mortality. The brain cannot produce glucose or other metabolic reserves, it depends on the supply of glucose, with insulin and glucagon as the role hormone in glucose control. [1, 10] In sepsis, hypoglycemia occurs due to the inability to break down glycogen reserves and hyperinsulinemia, on the other hand, metabolic changes in sepsis cause glucagon as a counter regulatory hormone failed to meet alternative glucose needs from other sources such as fatty acids and amino acids. Septicemia increased hormonal changes, pro-inflammatory cytokines, interleukins (IL-1, IL-6), and tumor necrosis factor (TNF)-alpha that cause hyperglycemia. [2-4]

Hypoglycemia can affect early brain development and contribute to neurological disorders such as cerebral palsy, mental retardation, blindness and intractable epilepsy. $[5,6]$ Prolonged hypoglycemia causes irreversible cellular dysfunction leading to organ failure and death. Sepsis and neonatal stress can also trigger hyperglycemia manifested by 
glucosuria and osmotic diuresis, leading to dehydration, ketosis, and metabolic acidosis. Hyperglycemia is a risk factor for intracranial bleeding that indirectly causes death. [7-9]

The purpose of this study was to prove the difference in mortality of neonatal sepsis with abnormal blood glucose compared to normal blood glucose. Researcher conducted a study by considering the mechanism of blood sugar homeostasis in newborns and analyzing confounding variables that had not been carried out in previous studies.

\section{Material and Method}

This study use retrospective cohort design study with span of January- August 2020. Subject of this study is neonate with 28-42 weeks of gestational age diagnosed with neonatal sepsis. Inclusion criteria were neonate with 28-42 weeks of gestational age diagnosed with neonatal sepsis confirmed from clinical sign or blood culture at neonatal ward Sanglah hospital. Exclusion criteria were neonatal sepsis with Diabetes Mellitus mother, neonatal sepsis with a suspected syndrome (endocrine or metabolic disorders), and incomplete medical record data. Subject is determined by taking the subject in sequence until the number of subjects is met.

Neonatal sepsis described as condition that fulfilled at least one major risk factor of infection or two minor risk factor of infections, accompanied by clinical signs and abnormal physical examination, with at least two positive laboratory studies (with or without positive blood culture results). Blood sugar levels described as blood sugar level checked when neonate already met the sepsis criteria. Blood sugar levels is carried out by the POC method using sample of capillary blood. This study divided blood sugar levels into (1) abnormal blood sugar levels, which are hypoglycemia (blood sugar levels less than $45 \mathrm{mg} / \mathrm{dL}$ ) and hyperglycemia (blood sugar levels $\mathrm{n}$ more than $145 \mathrm{mg} / \mathrm{dL}$ ), (2) normal blood sugar level or normoglycemia is the blood sugar level at $45-145 \mathrm{mg} / \mathrm{dL}$. Patients who met the previously mentioned inclusion and exclusion criteria were included in the study.

Data obtained from the sample, then collected and processed into SPSS, descriptive analysis used to describe the characteristics of research subjects based on outcome so that it can be assessed comparability between the two groups. The analysis to assess the association between variable blood glucose level and mortality was done by making a $2 \times 2$ cross-tabulation (dummy table). The statistical test used is chi-square test with the significance limit of 0.05 . All the variable were included in multivariate analysis. The analysis used is logistic regression, statistically significant with $95 \%$ confident interval $(\mathrm{CI})$ and $\mathrm{P}$ values $<0.05$.

\section{Result}

A total of 156 neonates participated in the study, 28 subjects were excluded because of incomplete medical record data or suspicion of a suspected syndrome. A total of 128 subject met the criteria, consisting of 64 subjects with normal sugar levels and 64 subjects with abnormal blood sugar levels. The data shows that male gender shows a higher mortality rate (37.8\%). The median gestational age of the study subjects was 33 weeks with a range of 28 to 37 weeks. Most of the gestational age was moderate to late preterm, but mortality occurred mostly at extremely preterm gestational age (71.4\%). The median birth weight of this study was 1900 grams with a range of 800 to 4010 grams, mortality was more common in birth weight under 1000 gram $(66.7 \%)$ and small for gestational age $(43.8 \%)$. Higher mortality found in subject with asphyxia $(45.3 \%)$. Mortality was greater in mechanically ventilated subjects $(58.1 \%)$. The duration of parenteral nutrition mostly less than 14 days but higher mortality found in duration more than 14 days (57.1\%). Early onset sepsis (90.6\%) and no growth blood culture $(84.4 \%)$ were majority in this study, mortality of positive blood culture and no growth was comparable. Characteristic of the sample were summarized in table 1 .

Bivariate analysis was conducted using chi square test indicated abnormal blood sugar level have higher mortality compared to normal blood sugar level. Distribution between mortality and blood sugar level were summarized in table 2 .

The difference of mortality in the abnormal blood sugar group is greater than the normal group, with a relative risk of 2.91 (95\% CI 1.61 to 5.25), $\mathrm{p}<0.001$. The differences in each variable that caused the incidence of death were identified by sub-analysis. Sub-analysis of the blood sugar level were summarized in table 3 .

The difference in the incidence of death in the hypoglycemia group was greater than in the normoglycemia group, with incidence of death in the hypoglycemia group was 3.75 times compared to the normoglycemia group, which was statistically significant with $p<0.001$. The incidence of death in the hyperglycemia group was greater than in the normoglycemia group, with incidence of death in the hyperglycemia group was 2.12 times compared to the normoglycemia group, which was statistically significant with $p$ 0.035. Multivariate analysis showed that in this study abnormal blood sugar level, mechanical ventilation and asphyxia have higher mortality in neonate with sepsis. Multivariat analysis summarized in table 4.

The results of the multivariate analysis showed that abnormal blood sugar levels increased the risk of death by 3.04 times $(95 \%$ CI 1.09 to $8.45, p$ 0.033). Multivariate analysis also found that the use of mechanical ventilation affected the incidence of death, with the result that neonates who received mechanical ventilation had a 12.33 times risk of death ( $95 \%$ CI 3.99 to $38.07, p<0.001)$. Asphyxia at birth also affected the incidence of death, namely 8.17 times the risk of experiencing death compared to birth without asphyxia (95\% CI 2.35 to $28.37, p<0.001)$. 
Table 1. Characteristic of Subjects.

\begin{tabular}{|c|c|c|c|}
\hline Characteristic & $\mathrm{N}=128$ & Death $N=43$ & Survivor, $\mathrm{N}=85$ \\
\hline \multicolumn{4}{|l|}{ Sex, N (\%) } \\
\hline \multirow[t]{2}{*}{ Male } & $82(64.1)$ & $31(37.8)$ & $51(62.2)$ \\
\hline & $46(35.9)$ & $12(26.1)$ & $34(73.9)$ \\
\hline \multicolumn{4}{|l|}{ Gestation age, $\mathrm{N}(\%)$} \\
\hline Extremely preterm & $7(5.5)$ & $5(71.4)$ & $2(28.6)$ \\
\hline Very preterm & $38(29,7)$ & $14(36.8)$ & $24(63.2)$ \\
\hline Moderate to late preterm & $41(32.0)$ & $13(31.7)$ & $28(68.3)$ \\
\hline Aterm & $42(32.8)$ & $11(26.2)$ & $31(73.8)$ \\
\hline \multicolumn{4}{|l|}{ Birth weight, N (\%) } \\
\hline ELBW & $15(11.7)$ & $10(66.7)$ & $5(33.3)$ \\
\hline VLBW & $28(21.9)$ & $10(35.7)$ & $18(64.3)$ \\
\hline LBW & $45(35.2)$ & $13(28.9)$ & $32(71.1)$ \\
\hline Normal birth weight & $40(31.2)$ & $10(25.0)$ & $30(75.0)$ \\
\hline \multicolumn{4}{|c|}{ Birth weight for gestational age, $\mathrm{N}(\%)$} \\
\hline Normal & $111(85.9)$ & $36(32.4)$ & $75(67.6)$ \\
\hline SGA & $16(12.5)$ & $7(43.8)$ & $9(56.2)$ \\
\hline LGA & $1(1.6)$ & $0(0.0)$ & $1(100.0)$ \\
\hline \multicolumn{4}{|c|}{ Mechanical ventilation, N (\%) } \\
\hline Yes & $62(48.4)$ & $36(58.1)$ & $26(41.9)$ \\
\hline No & $66(51.6)$ & $7(10.6)$ & $59(89.4)$ \\
\hline \multicolumn{4}{|l|}{ Parenteral nutrition, $\mathrm{N}(\%)$} \\
\hline$>14$ days & $14(10.9)$ & $8(57.1)$ & $6(42.9)$ \\
\hline$\leq 14$ days & $114(89.1)$ & $35(30.7)$ & $79(69.3)$ \\
\hline \multicolumn{4}{|l|}{ Asphyxia } \\
\hline Yes & $75(58.6)$ & $34(45.3)$ & $41(54.7)$ \\
\hline No & $53(41.4)$ & $9(17.0)$ & $44(83.0)$ \\
\hline \multicolumn{4}{|l|}{ Type of sepsis N (\%) } \\
\hline Early onset sepsis (EOS) & $116(90.6)$ & $39(33.6)$ & $77(64.6)$ \\
\hline Late onset sepsis (LOS) & $12(9.4)$ & $4(33.3)$ & $8(66.7)$ \\
\hline \multicolumn{4}{|l|}{ Blood culture, N (\%) } \\
\hline Positive & $20(15.6)$ & $7(35.0)$ & $13(65.0)$ \\
\hline No Growth & $108(84.4)$ & $36(33.3)$ & $72(66.7)$ \\
\hline
\end{tabular}

Table 2. Distribution between blood sugar level and mortality.

\begin{tabular}{llllll}
\hline & Death (n=\%) & Survivor (n=\%) & RR & CI95\% & p \\
\hline Abnormal blood sugar level & $32(50.0)$ & $32(50.0)$ & 2.91 & $1.61-5.25$ & $<0.001$ \\
Normal blood sugar level & $11(17.2)$ & $53(82.8)$ & & & \\
\hline
\end{tabular}

Table 3. Subanalysis of blood sugar level with mortality.

\begin{tabular}{|c|c|c|c|c|c|}
\hline & Death, N (\%) & Survivor, N (\%) & $\mathbf{R R}$ & CI95\% & $\mathbf{P}$ \\
\hline Normoglycemia & $11(17.2)$ & $53(82.8)$ & & & \\
\hline Hypoglycemia & $20(64.5)$ & $11(35.5)$ & 3.75 & $2.06-6.82$ & $<0.001$ \\
\hline Hyperglycemia & $12(36.4)$ & $21(63.6)$ & 2.12 & $1.04-4.26$ & 0.035 \\
\hline
\end{tabular}

Table 4. Multivariate Analysis Factors Associated in LOS of SAM.

\begin{tabular}{lllll}
\hline Variable & SE & RR & CI 95\% & $p$ \\
\hline Abnormal blood sugar & 0.52 & 3.04 & $1.09-8.45$ & 0.033 \\
Mechanical ventilation & 0.58 & 12.33 & $3.99-38.07$ & $<0.001$ \\
Asphyxia & 0.64 & 8.17 & $2.35-28.37$ & 0.001 \\
Male & 0.55 & 2.28 & $0.78-6.64$ & 0.131 \\
Parenteral nutrition >14 days & 0.69 & 0.69 & $0.18-2.70$ & 0.594 \\
Birth weight (ELBW) & 0.77 & 0.95 & $0.21-4.33$ & 0.947 \\
Gestational age Extremely preterm & 0.72 & 1.01 & $0.25-4.10$ & 0.991 \\
Birth weight for gestational age (SGA) & 0.69 & 1.19 & $0.31-4.54$ & 0.809 \\
Type of sepsis (EOS) & 1.02 & 0.24 & $0.32-1.78$ & 0.162 \\
Positive blood culture & 0.74 & 0.49 & $0.11-2.10$ & 0.334 \\
\hline
\end{tabular}

\section{Discussion}

The subjects of this study were neonates with a gestation age of 28-42 weeks with sepsis, with total sampling 128 neonate. Neonatal sepsis is one of the leading causes of mortality in neonates, especially in preterm infants and LBW $[11,13]$.

Characteristic data show that mortality in neonates with sepsis occurs more frequently at moderate to late preterm 
gestation, mortality in neonates with sepsis was more common in LBW, with the median birth weight of the study subjects being 1900 grams. The results of this study are in accordance with previous research, from 138 cases of neonatal sepsis, $28.3 \%$ of the deaths occurred, where LBW (RR $8.495 \%$ CI $(2.4-29.0), p<0.001)$ and prematurity (RR 3.4 95\% CI $(1.0-11.0), \quad p=0.042)$ were significantly associated with mortality in neonatal sepsis [11].

Most of the subjects were born with asphyxia (58.6\%), the most common type of sepsis was SNAD (90.6\%) with the highest blood culture was no growth $(84.4 \%)$. In tis study found that mortality in subjects with positive culture results was obtained with $S$. hemoliticus. This result show different results from other study that out of 351 neonates with sepsis, positive blood cultures occurred in $47.6 \%$ of the subject. The study also found that the most mortality occurred in E. coli, Coagulase negative staphylococus, S. aureus and Klebsiella infections [12].

Characteristic data showed more mortality in subjects with mechanically ventilated and given total parenteral nutrition for more than 14 days, $58.1 \%$ and $57.1 \%$, respectively. Milstone et al. (2013) showed that infections that could occur in blood vessel access due to parenteral nutrition or central line associated blood stream infections (CLABSI) increased after more than two weeks. This infection can increase the mortality rate in neonates with sepsis. The study, obtained from 4797 access attachments for parenteral nutrition, there were 149 incidents of CLABSI [13-15].

Sepsis is known to be the cause of hypoglycemia in $9.6 \%$. The increase in stress hormones such as adrenaline, cortisol and glucagon in neonatal sepsis raises blood sugar levels in sepsis. Neonates with sepsis accompanied by hyperglycemia or hypoglycemia have a high risk of death [1].

In this study, mortality found in $32(50 \%)$ subjects with abnormal sugar levels and $11(17.2 \%)$ subjects with normal sugar levels. Similar results were found in the study by Sabhzei et al. (2014) that mortality in neonates with abnormal blood sugar levels was $50.84 \%$ [16].

In the study, the most abnormal sugar levels found in neonatal sepsis were hyperglycemia $(51.6 \%)$, but mortality occurred more in hypoglycemia (RR 3.75 (95\% CI 2.06 to 6.82 ) with $p<0.001$. Research by Islam et al. (2016) found a similar result that the majority of abnormal blood sugar was hyperglycemia (15.3\%) compared to hypoglycemia (13.45\%). Mortality was more common in neonatal sepsis with hypoglycemia $(42,8 \%)$ compared to normoglycemia $(10.8 \%)$ although not significantly significant $(p>0.05)$. Neonates with sepsis having problems with nutrition, increased metabolic requirements, and hypothermia, all these condition increase the risk of hypoglycemia. Ahmad et al. (2012) found different results, that in neonates with sepsis followed by positive blood culture, mortality occurred in $9.9 \%$ of patients with blood sugar $40-100 \mathrm{mg} / \mathrm{dl}, 23.2 \%$ at blood sugar between 101-200 $\mathrm{mg} / \mathrm{dl}$, and $48.6 \%$ at blood sugar level $>200 \mathrm{mg} / \mathrm{dL}$ [1].

Hypoglycemic sugar levels can be a form of metabolic adaptation during the extrauterine phase, sugar levels below $30 \mathrm{mg} / \mathrm{dl}$ can occur in term neonates at 3 hours early life and only $9 \%$ of them require therapy. Prolonged hypoglycemia can be life threatening, this occurs in neonatal sepsis where sugar levels can fall below $40 \mathrm{mg} / \mathrm{dl}$ and increase the incidence of mortality. Neonates with hypoglycemia have a higher risk of receiving ventilation assistance, so that it can be a prognostic marker of neonatal sepsis [17].

In this study the mortality rate for neonatal sepsis in hyperglycemia was $36.4 \%$ and normoglycemia was $17.2 \%$ (RR 2.12 (95\% CI 1.04 to 4.26$)$ with a value of $p=0.035$. Research by El-Mekkawy and Ellahony (2019) showed similar result, hyperglycemia was found in $24.6 \%$ of neonates treated at the NICU and $78.8 \%$ of them were caused by sepsis. Hyperglycemia was also found to increase mortality compared to normal sugar levels in neonatal sepsis and indirectly indicates the severity of a sepsis, so it can be an indicator of mortality in neonatal sepsis $[18,19]$.

The multavariate analysis found that mortality was also influenced by asphyxia (RR 8.17 (95\% CI 2.35 to 28.37 ) with $p=0.001$, and the use of mechanical ventilation (RR $12.33(95 \%$ CI 3.99 to 38,07$)$ with $p<0.001$. Hossain et al. (2009) found mortality in neonates with neonatal sepsis was $70.6 \%$, mortality was also high in neonates with birth weight $<2500$ grams and gestational age $<34$ weeks $(\mathrm{p}<0.005)$. In ventilated neonates, there is also an impaired acid-base balance with a blood $\mathrm{pH}<7.3$, which increases the mortality rate [20].

The degree of asphyxia can also affect the decrease in sugar levels resulting in poor outcome. When asphyxia occurs, anaerobic glycolysis increases lactic acid production. A state of severe acidosis can indirectly increase glycolysis and cause impaired cardiovascular function. Hypoxia that occurs also increases consumption of glucose, the combination of hypoglycemia and asphyxia increases mortality in neonatal sepsis [21-23].

Limitation of this study is we measure blood sugar level from peripheral blood samples. Venous blood samples can provide more accurate information on blood sugar levels in the body. Blood sugar levels was taken when neonate diagnosed with sepsis so that it cannot detect episodes of changes in blood sugar levels earlier.

\section{Conclusion}

This study proves that abnormal blood sugar levels increase mortality in neonatal sepsis. Multivariate analysis also showed that the use of mechanical ventilation and asphyxia at birth increased mortality in neonatal sepsis.

In neonatal sepsis blood sugar should be monitored continuously so that abnormal blood sugar level can be treated immediately. This study also found that the use of mechanical ventilation and asphyxia affected mortality, so it is recommended to do a further research to find relationship between mortality with each variable.

\section{Disclosure}

All the authors do not have any possible conflicts of interes. 


\section{References}

[1] Aklima, J., Islam, M. Z., Yesmin., Islam, M. S., Chakma, K., Alauddin, M., Bhuiyan, R. H. 2017. Evaluation of hypoglycemic status and causative factors in neonatal sepsis. Int J Contemp Pediatr; 4 (6): 1927-1933.

[2] Ahmad, S., Khalid, R. 2012. Blood glucose levels in neonatal sepsis and probable sepsis and its association with mortality. Journal of the College of Physicians and Surgeons Pakistan; 22 (1): 15-18.

[3] Gentile, L. F., Nacionales, D. C., Lopez. M. C. 2014. Protective immunity and defects in the neonatal and elderly immune response to sepsis. J Immunol; 192 (11): 3156-3165.

[4] Gupta, B. K., Gupta, B. K., Srivastava, A. K., Chetri, P. 2019. Glycemic status and its effect in neonatal sepsis - A prospective study in a tertiary care hospital in nepal. $J A d v$ Pediatr Child Health; 2: 15-19.

[5] Thompson-Branch, A., Havranek, T. 2017. Neonatal hypoglycemia, Pediatrics in Review; 38 (4): 147-155.

[6] Beardsall, K., Vanhaesebrouck, S., Ogilvy-Stuart, A. L., Vanhole, C., Palmer C. R., Ong, K., Weissenbruch, M., Midgley, P., Thompson, M., Thio, M., Cornette, L., Ossuetta, I., Iglesias, I., Theyskens, C., de Jong, M., Gill, B., Ahluwalia, J. S., Zegher, F., Dunger, D. B. 2010. Prevalence and determinants of hyperglycemia in very low birth weight infants: Cohort analyses of the nirture study, J Pediatr; 157: 715-719.

[7] Shaha CK, Dey SK, Shabuj KH, Chisti J, Mannan, Jashimuddin, dkk. Neonatal sepsis a review. Bangladesh $J$ Child Health. 2012; 36 (2): 82-9.

[8] Branch, A. T., Havranek, T. 2017. Neonatal hypoglycemia. Pediatric in Review; 38 (4): 147-155.

[9] Yadav, D. 2016. Persistent neonatal hypoglycaemia. SM Group; 5: $1-15$.

[10] Boghossian, NS., Geraci, M., Edwards, EM., Horbar, JD. 2018. Morbidity and mortality in small for gestational age infants at 22 to 29 weeks' gestation. PEDIATRICS; 141 (2): 1-10.

[11] Kardana, M., Artana, D., Junara, P., Sukmawati, M. 2017. Sepsis neonatorum. In: Arhana, B. N. P., Ariawati, K., Gustawan, I. W., Yantie, N. P., editor. Pedoman Praktis Klinis Ilmu Kesehatan Anak. $1^{\text {st }}$ Ed. Denpasar: RSUP Sanglah. p. 418-421.

[12] Turhan, E. E., Gürsoy, T., Ovalı, F. 2015. Factors which affect mortality in neonatal sepsis. Turk Pediatri Ars; 50: 170-175.

[13] Milestone, A., Reich, N., Advani, S., Yuan, G., Bryant, K., Coffin, S., Huskin, C., Livingston, R., Smith, B., Song, X. 2013. Catheter dwell time and CLABSI in neonates with PICC: A multicenter cohort study. Pediatrics; 132 (6); 1609-1615.

[14] Jung, C. H., Cho, H. K. 2019. Central line-associated bloodstream infections in neonates. Korean J Pediatri; 3: 79-84.

[15] Calkins, K., Venick, R. S., Devaskar, S. U. 2014. Complications associated with parenteral nutrition in the neonate. Clin Perinatol; 41 (2): 331-345.

[16] Sabzehei, M. K., Afjeh, S. A., Shakiba, M., Alizadeh, P., Shamshiri, AR., Esmaili F. 2014. Hyperglicemia in VLBW infants, incidence, risk factors, and outcome. Arch Iran Med; 6: 429-433.

[17] Hawkes, C. P., Stanley, C. A. 2017. Pathophysiology of neonatal hypoglycemia. In: Hockenberry, MJ., Rhodes, K., Wilson, D., editor. Fetal and Neonatal Physiology: Maternal Child Nursing Care. $6^{\text {th }}$ Ed. Philadelphia: Elsevier. p. 1552-1562.

[18] El-Mekkawy, M., Ellahony, D. M. 2019. Prevalence and prognostic value of plasma glucose abnormalities among full-term and late-preterm neonates with sepsis. Egyptian Pediatric Association Gazette; 67 (2): 1-7.

[19] Hay, W. W., Rozance, P. J. 2018. Neonatal hyperglycemia, causes, treatments, and cautions. The Journal of Pediatrics; 2 : 1-3.

[20] Hossain, M., Shirin, M., Al Mamun, MA., Hasan, N., Sahidullah. 2009. Predictors of mortality in ventilated neonates in intensive care unit. Bangladesh J Child Health; 33 (3): $77-82$.

[21] Mufidati, L., Anggraini, A., Wibowo, T. 2017. Asphyxia as a risk factor for neonatal hypoglycemia. J Nepal Paediatr Soc; 37 (2): 111-116

[22] Iqbal, Q., Younus, M., Ahmad, I., Iqbal, J., Charoo, Ali, S. 2015. Neonatal mechanical ventilation: Indication and outcome. Indian J Crit Med; 19 (9): 523-527.

[23] Islam, M. S., Mis, M. A. H., Rokshanaakhter, K., Haque, P., Malik, M. A, 2016. Glycemic status and its effect in neonatal sepsis in a tertiary care hospital. Bangladesh J Child Health; 40 (1): 21-27. 